\title{
Mutualisation des expériences dans l'enseignement des langues dans les IUFM
}

Sabine Ulrich

\section{OpenEdition}

1 Journals

Édition électronique

URL : http://journals.openedition.org/trema/1463

DOI : 10.4000/trema.1463

ISSN : 2107-0997

Éditeur

Faculté d'Éducation de l'université de Montpellier

\section{Édition imprimée}

Date de publication : 1 janvier 2003

Pagination : 161-175

ISSN : 1167-315X

\section{Référence électronique}

Sabine Ulrich, « Mutualisation des expériences dans l'enseignement des langues dans les IUFM », Tréma [En ligne], 20-21 | 2003, mis en ligne le 01 avril 2003, consulté le 04 mai 2019. URL : http:// journals.openedition.org/trema/1463; DOI : 10.4000/trema.1463

Ce document a été généré automatiquement le 4 mai 2019.

Trema 


\title{
Mutualisation des expériences dans l'enseignement des langues dans les IUFM
}

\author{
Sabine Ulrich
}

\section{Problématique de formation dans laquelle s'insère ce projet}

1 La place relative de l'enseignement des langues s'est accrue dans le système scolaire français depuis quelque temps. La sensibilisation aux langues en primaire est devenue un apprentissage, la notion de niveau seuil en langues, qui existait dans les années 1970, est réapparue sous la forme des niveaux du cadre européen commun de référence pour les langues publié par le Conseil de l'Europe servant de référents à la fois dans la formation continue des enseignants mais aussi dans l'apprentissage des enfants. Les concours pour les professeurs de lycée et collège ont vu le coefficient de l'épreuve de didactique augmenté.

Tous ces éléments concourent à montrer que les langues sont un enjeu majeur dans l'enseignement aujourd'hui et ont présidé à la proposition et à l'organisation du séminaire PSE (Pôle Sud-Est) qui s'est déroulé à l'IUFM de Montpellier les 24 et 25 avril derniers.

Des thèmes forts furent dégagés et proposés dans les différents IUFM pour que les formateurs intéressés par ce projet puissent aisément s'y inclure.

\section{Les thèmes forts de ce séminaire}

\subsection{La notion de genre}

2 Nous reprenons ci-dessous le texte de l'intervention de Mireille Baurens, formatrice à l'IUFM de Grenoble. 


\subsubsection{Inégalités, équité, éthique ou pour un plaidoyer de la variable genre}

3 Les filles ont des résultats scolaires supérieurs à ceux des garçons, partout. Elles sont plus nombreuses à réussir le bac. Les garçons, une fois jeunes adultes, surpassent, grâce à leur bagage scolaire, leurs compagnes, en terme de poursuite d'études, de carrières socioprofessionnelles, de salaires, d'investissement politique, de reconnaissance historique...

Le contraste est effarant entre le nombre de carrières investies par les femmes et par les hommes. On constate que l'invisible "plafond de verre ", auquel les femmes se heurtent lors de leur carrière professionnelle, existe même dans le secteur extrêmement féminisé et public qu'est l'enseignement.

Ce qui s'inscrit comme performances contrastées à l'école au départ, et dont pâtiraient les garçons, s'inverse totalement dans le temps et se transcrit aux dépens des filles, à la sortie de l'école. La scolarité que vivent les filles et les garçons n'est pas la même.

Le postulat est donc le suivant: au sein de ce qui est visible à l'école (en particulier les différences de performance entre filles et garçons) se tisse une trame, invisible tout d'abord, qui fabrique, d'une part des comportements et des représentations discriminantes envers les filles et d'autre part, une résistance et une combativité propres aux garçons.

5 C'est du maintien des ségrégations entre filles et garçons au terme et au cœur de leur scolarité qu'il faut s'informer, pour pouvoir les réduire. Dans ce but, il existe 8 éléments à prendre en compte :

- les professeur(e)(s) s'occupent davantage, quantitativement et qualitativement, de leurs élèves garçons que de leurs élèves filles ;

- le déséquilibre existe dans les programmes et les matériaux scolaires où on observe que les femmes et filles sont largement exclues ou stéréotypées ;

- le sexe de l'élève joue sur l'évaluation et les exigences pédagogiques (existence d'un double standard) ;

- la pédagogie de l'encouragement n'est pas constante envers les filles ;

- l'impact des représentations stéréotypées des jeunes est important sur la réussite scolaire ;

- l'orientation reflète cette discrimination ;

- un quasi absolu manque de conscience de cet état de fait existe du côté des enseignant(e)(s) qui n'y apportent donc aucune vigilance particulière ;

- les paradoxes sexués de la mixité, mise en place en fait, pour répondre à des urgences démographiques, sans réflexion pédagogique préalable.

Une définition se profile maintenant, après ce tableau sur les inégalités de sexe.

On parle de genre, par opposition au sexe biologique, pour mettre en exergue ce que la culture crée comme différenciation : d'un côté les facteurs de l'inné, minimes et de l'autre les résultats de la socialisation, en particulier par l'école, pour ce qui nous intéresse ici.

7 Il faut apprendre à intégrer le genre, à penser le genre, comme variable à prendre en compte dans les observations et les pratiques de classe. Il faut, selon les propos tranchés de Annick Durand-Delvigne ${ }^{1}$ " faire travailler la mixité, cela veut dire utiliser explicitement et pragmatiquement la mixité comme révélateur des pratiques et des interactions entre élèves et enseignant(e)(s) marqués par un système catégoriel de sexe asymétrique afin d'asseoir plus solidement une pédagogie antisexiste." 


\subsubsection{Quel rôle pour les langues dans la mission d'équité entre les sexes ?}

\section{Quelques réalisations contextualisées, en anglais}

8 Les interactions nombreuses propres à la classe de langue dessinent un espace de relations de domination facile si l'on n'exerce pas une vigilance particulière. Les langues sont donc un révélateur de cette situation. Choisir les langues comme domaine de recherche est lié à plusieurs facteurs :

- il s'agit d'un choix didactique ;

- mais aussi d'un choix éthique : l'ouverture à l'Autre, à l'autre culture, commence avec le respect de l'autre entre autre, de l'autre sexe ;

- enfin, d'un choix d'angliciste dans la mesure où les recherches anglophones ont largement précédé les nôtres sur la question des sexes à l'école.

9 Il a donc fallu « vérifier » l'hypothèse selon laquelle les cours de langue pourraient jouer un rôle important dans la mission d'équité de l'école. Les expériences suivantes ont été tentées :

- De nombreuses observations de cours, filmés, confirment ce que la recherche souligne dans d'autres disciplines.

- Des entretiens avec des professeurs de langues, confirment la réticence des un(e)(s) et des autres à réaliser l'existence de cette ségrégation entre filles et garçons à l'école.

- La mise en place d'un atelier de mémoires professionnels pour les professeur(e)(s) d'école où je propose d'intégrer la variable du genre dans leur réflexion didactique. L'atelier confirme la nécessité de sensibiliser : en regardant, en réfléchissant, on peut comprendre ce qu'il se passe et influer sur ses propres pratiques.

Lors des premiers entretiens, les enseignant(e)(s) réitèrent leur assertion de neutralité, ne se sentent pas concernés : qui en effet adopterait des pratiques discriminatoires de façon consciente?

Puis, par la suite, c'est la découverte. Elles et ils affichent une extrême surprise, semblent prendre conscience de cette réalité et en général, agissent, pour modifier leur pratique. Ainsi, cette année, plusieurs jeunes professeur(e)(s) d'école, devant la vidéo de leurs propres cours, ont été médusées : elles ont orienté leur travail de mémoire sur la mixité, compromise dans leurs interventions en cours de langue, de par l'assiduité que les enseignantes devaient prodiguer aux garçons extrêmement agités.

\subsubsection{Pour conclure, de façon temporaire...}

11 Rendre visible l'invisible? Prendre conscience des inégalités entre filles et garçons que l'on encourage, ou du moins auxquelles on contribue sans s'en apercevoir, parce que l'on ne s'en aperçoit pas... C'est par la prise de conscience que l'on pourra les réduire, du moins en partie.

Il faut que chacune et chacun, nous nous sentions investi(e)(s) et responsables afin que s'épanouissent la prise en compte d'individus sexués et la mise en place de pratiques liées à la coopération, et non à la compétition, entre les sexes.

12 La prise de conscience chez les enseignant(e)(s) est un indispensable palier. La formation doit aller à la rencontre puis à l'encontre de leur conviction de la neutralité à l'œuvre dans leur pratique. En réalité, ce qui se passe est très sexué. Il est nécessaire d'avoir des outils d'auto-observation pour s'en rendre compte. 
Ensuite, une distance critique par rapport aux matériaux didactisés et leur possible sexisme est aisée à partir des études déjà réalisées. Elle doit s'assortir de la valorisation des femmes dans les enseignements dispensés.

\section{2. Étude comparative de deux systèmes de formation des enseignants}

13 Le système britannique et le système français : cette communication a été présentée par Shirley Lawes (Université de Londres) d'après son travail de recherche.

\subsubsection{Les premières mesures sous le gouvernement de $\mathrm{M}^{\mathrm{me}}$ Thatcher}

La sélection des futurs enseignants, à l'époque, était faite au niveau licence et sur dossier. Elle était opérée par un Directeur-Tuteur, qui assurait le partenariat établissement scolaire - université (la formation des futurs enseignants en Grande-Bretagne étant faite par l'université.) Les formateurs devaient avoir au minimum un Master's Degree.Ils devaient pouvoir aussi faire état d'une compétence en pratique professionnelle.

Le tuteur s'occupait de la plus grande partie de la formation. Il était assisté par des professeurs de l'Université. Les futurs enseignants devaient assurer une formation pratique (block practice)qui allait en progressant au cours de la formation jusqu'à atteindre $50 \%$ de pratique sur le terrain.

Il y avait trois visites pendant l'année qui étaient toutes des visites formatives. La formation se composait de 550 heures de cours en présentiel et des séances pratiques dans les établissements.

\subsubsection{En 1997, changements politiques avec le Gouvernement de T. Blair}

16 La seule réforme dans la formation des enseignants est le National Curriculum, une liste de 87 compétences standards que doivent acquérir les futurs enseignants en formation initiale et qui établit une politique commune pour la formation des enseignants dans tout le pays. Les compétences listées dans ce document sont toutes de conception assez behaviouristes.

Le National Curriculumnécessite d'être au courant de ce qui se passe en amont et en aval dans le développement du collégien - lycéen (développements psychologique, physique...) Il faut également posséder des compétences en TIC qui font l'objet d'un examen spécifique. Enfin, il est nécessaire d'être au fait des stratégies visant à promouvoir l'apprentissage, etc...

18 L'objectif essentiel est l'obtention du National Curriculum :il s'agit donc de faire acquérir aux futurs enseignants les compétences listées dans ce document de façon à ce qu'ils puissent « obtenir » le National Curriculumet être considérés aptes à enseigner. Ce régime est très prescriptif, très différent de celui observé en France et bien plus orienté vers les savoir-faire que vers les savoirs. La partie théorique de la formation a été très marginalisée.

19 C'est pourquoi, actuellement, des théoriciens et didacticiens préconisent un retour à la formation théorique car elle permet d'assurer la qualité et le renouvellement de la pratique. Mais, dans la réalité, la nécessité de parvenir aux "standards"l'emporte sur toute 
autre considération. L'attitude en situation l'emporte sur le savoir. C'est la conformité aux standards qui prime.

- Formation Générale Disciplinaire (180 heures).

- Group introduction

- Initiation à une langue inconnue (Arabe, par exemple).

- Les langues à l'école.

- Les modèles d'apprentissage et l'histoire des langues, etc..

Il y existe deux types de formations :

- la formation en université qui a été décrite ci-dessus ;

- le PGC (Post Graduate Certification)qui est ouvert aux plus de 25 ans.

21 La formation se fait sur place, dans un établissement où l'on a été recruté. Le futur enseignant est payé et fait $80 \%$ de l'horaire officiel. Il a un maître de stage qui assure le suivi de sa formation. L'université garantit la qualité de l'ensemble. Les "induction years"(1 re année d'exercice) existent aussi en Grande-Bretagne, avec des résultats variables.

Toutes les activités proposées dans ces formations doivent avoir un rapport avec les compétences attendues (les différents modèles d'enseignement, visionnement de vidéos sur l'approche communicative, repérages de la bonne pratique dans des enseignements filmés...) ; les prescriptions pour l'enseignant relèvent plus du manuel que de la formation théorique.

\subsubsection{Conclusions}

La liste des thèmes abordés dans la formation disciplinaire montre une ressemblance avec le contenu de la formation en France bien qu'il y ait des thèmes abordés (la formation générale et commune) qui apparaissent dans la formation disciplinaire en GrandeBretagne.

La pratique réflexive constitue l'orthodoxie en Grande-Bretagne. Les concepteurs de la formation et les formateurs eux-mêmes pensent que sans l'apport des Sciences de l'Éducation, ils n'ont pas assez de bases pour former un professionnel de l'éducation.

Néanmoins, la conception qui préside à la formation en France est que la théorie aussi est nécessaire, mais que son acquisition demande du temps. Ceci peut apparaitre paradoxal et contradictoire, puisque les stagiaires demandent des modèles, des standards. Or, nous savons tous que sans formation, il n'y a pas de recul épistémologique.

\subsection{Généralisation de l'enseignement des langues vivantes à l'école élémentaire}

\section{Vers une nouvelle conception du métier et de la formation des professeurs des écoles ? Communication présentée par Marie-Christine Deyrich et Sabine Ulrich (IUFM de Montpellier).}

La formation au « métier » d'enseignant est d'autant moins une formation professionnelle comme les autres qu'elle suppose l'intégration fréquente d'éléments nouveaux venant transformer les modèles de professionnalité établis ${ }^{2}$ et, partant, les besoins de formation qui en découlent. Ainsi, la généralisation de l'enseignement des langues vivantes étrangères à l'école nous invite-t-elle à mettre en perspective, d'une part, les attentes institutionnelles concernant la professionnalité du professeur des écoles enseignant une 
langue vivante (désormais LV) et, d'autre part, la façon dont les professeurs-stagiaires conçoivent l'intégration de compétences inhabituelles dans leur identité professionnelle.

\subsubsection{L'intégration de la LV dans l'enseignement des PE}

Que disent les programmes et orientations pédagogiques?

- Les termes le plus souvent associés à cet enseignement sont : « un enseignement comme les autres ".

- Néanmoins les «spécificités de l'enseignement de la LV » sont également mentionnées.

- Enfin dans les textes et orientations les plus récents apparait la mention des «spécificités de l'enseignement de la LV en primaire ».

Mais on sait qu'il existe des pré-requis pour la formation en langues :

- de solides connaissances disciplinaires ;

- une conception de ce qu'est la langue et de son apprentissage.

Enfin, l'objectif de la formation est d'amener nos stagiaires à devenir des praticiens réflexifs, il faut donc que nos stagiaires soient également capables :

- de décentration, d'avoir une « pensée didactique »;

- d'assurer la gestion complète de la transposition didactique.

Ces éléments ont orienté nos interrogations sur le profil de l'enseignant qu'il convient de viser : technicien et/ou praticien réflexif, généraliste polyvalent et/ou spécialiste précis de l'enseignement de la LV à l'école élémentaire?

Pour examiner comment les modifications de la teneur de l'exercice professionnel sont envisagées sur le terrain, nous avons sollicité le point de vue des formés. Un premier questionnaire a été donné aux professeurs-stagiaires au tout début de leur formation, un second en janvier.

\subsubsection{Bilan du premier questionnaire}

31 Ce questionnaire a révélé la nécessité d'une construction identitaire. La question posée aux stagiaires était :

\section{Qui peut/doit enseigner les langues vivantes à l'école ?}

$95 \%$ d'entre eux ont répondu que l'enseignement devait être assuré par un intervenant extérieur. Sur ces $95 \%, 88 \%$ ont ajouté que cet enseignement devait être assuré par un collègue PLC.

Ces réponses nous ont amenées à l'hypothèse que l'enseignement des LV ne faisait partie ni de la culture ni de l'identité professionnelles de nos stagiaires. En effet, cet enseignement a été introduit relativement récemment dans la formation initiale des PE et ils n'ont pas «vécu » cet enseignement à l'école primaire: cette discipline n'était pas enseignée quand ils étaient élèves.

Dès lors la construction de référents de professionnalité était nécessaire. Nous avons estimé qu'ils devaient être basés à la fois sur des savoirs et des savoir-faire :

- dans la langue (compétence de communication);

- sur la langue (compétence métalinguistique); 
- culturels (compétence culturelle).

À ce stade de la réflexion, il fallait avoir une vision plus claire des représentations des stagiaires sur :

- la langue et de son apprentissage ;

- l'enseignement de la langue.

Ces priorités ont guidé l'élaboration du second questionnaire.

\subsubsection{Bilan du deuxième questionnaire et conclusion}

L'analyse des réponses à ce questionnaire met l'accent sur trois points importants à prendre en compte dans la formation des PE à l'enseignement des LV à l'école primaire :

- Il faut un bouleversement des représentations de telle sorte que le professeur des écoles considère qu'il doit aussi enseigner une langue vivante au même titre que les autres matières.

- Il faut changer les conceptions actuelles sur l'enseignement des langues dans le primaire : il doit s'agir d'une matière d'enseignement-apprentissage à part entière.

- L'objectif d'apprentissage (s'inscrivant dans une progression) doit primer sur tout le reste et donc il faut donner les moyens aux futurs enseignants d'agir sur la transposition didactique, donc sur les contenus.

Cependant, les contradictions et les décalages observés entre les ambitions affichées, la perception de l'identité professionnelle des professeurs des écoles et les possibilités offertes, tant en formation initiale que continue, nous incitent à la prudence. C'est pourquoi nous soutenons qu'il est illusoire d'imaginer que le " greffage » de la discipline langue vivante sur une professionnalité «ancienne ${ }^{3}$ » pourra aller de soi si l'on ne se donne pas les moyens pour un travail en profondeur.

\subsection{Un exemple de classe linguistique à Frontignan (Hérault)}

\section{Expérience présentée par Didier Baumlé, animateur en langue, académie de Montpellier.}

À la lecture des nouvelles orientations dans l'enseignement des LV, il y a eu volonté, de la part des animateurs en langues de l'Hérault, de proposer une classe d'immersion linguistique en anglais de trois jours. Une école de Frontignan a accepté ce projet et une vidéo de 12 minutes a été réalisée. Il s'agissait d'une expérience intra-muros,c'est-à-dire faite au sein même de l'école. Les 52 élèves concernés avaient commencé l'apprentissage de l'anglais en début d'année scolaire. Les participants étaient :

- le directeur de l'école Marcel Pagnol : J. Facoltoso ;

- les animateurs/trices de langues du département : Didier Baumlé, Anne Igual, Catherine Judell-Dufour, Karine Mira ;

- les enseignants : Guillaume Fritschy (PE2), Gilles Capodanno ;

- les intervenantes extérieures : Laurence Parsegian, Bénédicte Pastourel, Marjorie Chevalier ;

- les assistantes : Anna Fitzgerald, Mari Myhre.

L'objectif de cette classe linguistique était de produire un spectacle pour fédérer tous les élèves. Il n'y a pas eu de projet pédagogique précis car cette expérience aurait dû se dérouler extra-muroset les locaux prévus n'ont pas été disponibles au dernier moment. Le 
projet pédagogique prévu pour l'organisation initiale ne pouvait fonctionner dans la nouvelle organisation. Un projet a donc été élaboré au soir de la première journée.

L'atout de cette formation a été que par ce biais, tous les élèves ont eu la possibilité de profiter d'un bain de langue quel que soit le niveau social des familles. De plus, l'idée de départ était d'impulser quelque chose. Or, cette expérience a été un déclencheur puisque l'IEN a été partie prenante du projet.

L'organisation et la réalisation de cette classe linguistique nous ont permis de constater que sept points sont vraiment importants dans l'élaboration de ce type de projets :

- le lieu : possibilité de séjours extra-muros,voire à l'étranger... ;

- la durée ;

- le financement;

- le public ;

- le contenu pédagogique : ateliers, modules ;

- l'encadrement ;

- les partenaires.

En conclusion, on peut espérer que si ce type d'expériences est présenté aux PE2, peutêtre se sentiront-ils moins démunis en ce qui concerne l'enseignement d'une langue vivante. On peut enfin se demander s'il ne serait pas intéressant d'intégrer cela à la formation des stagiaires.

\subsection{Le mémoire PLC2}

Communication présentée par Marie-Hélène Clavères (IUFM de Montpellier.).

\subsubsection{Présentation de l'expérience}

L'expérience qui est décrite ici a été menée dans l'académie de Montpellier sur les deux sites qui assurent la formation PLC en anglais : Montpellier et Perpignan. Les conclusions présentées sont basées sur l'analyse d'une cinquantaine de mémoires par an depuis 1992. Cette expérience commença donc un an après la création des IUFM. Les formateurs de l'époque n'étaient pas du tout satisfaits des mémoires présentés. Les stagiaires présentaient un projet pédagogique personnel qui échappait à toute forme d'évaluation rationnelle.

Progressivement, les formateurs ont reconsidéré l'architecture de la formation PLC et se sont rendus compte que sous le chapeau didactique, se trouvaient beaucoup d'items concernant l'enseignement de la langue et les stagiaires n'entendaient parler d'apprentissage que lors des formations générales et communes et des apprentissages non linguistiques. Donc, ils ont décidé de lier le mémoire à la notion d'apprentissage.

Par conséquent, la consigne donnée aux stagiaires en début d'année de formation fut de prendre comme point de départ un corpus de productions d'élèves. Mais, il était difficile de collecter des énoncés erronés car il fallait les entraîner à trouver des protocoles de collectes. Cet entraînement a été basé sur les travaux de Bronckart. On a fourni aux stagiaires une bibliographie dans laquelle l'ensemble des ouvrages donne des exemples de protocoles d'expérimentation. À partir de là, les stagiaires construisent des corpus analysables.

De plus, il semblait utile qu'à un moment de leur carrière les stagiaires soient confrontés 
à la recherche sur la didactique des langues née en Europe, qu'ils sachent qu'il existe un mouvement international dans ce domaine là.

Le troisième objectif de cette expérience était que les stagiaires considèrent l'erreur autrement. Nous avons considéré que former des stagiaires consiste bien sûr à les préparer à enseigner mais aussi à leur donner l'occasion de faire des choses qu'ils n'auront plus l'occasion de faire, par exemple, considérer l'erreur positivement.

Très vite, cela s'est élargi aux rapports entre les productions verbales des élèves et les représentations métalinguistiques. En effet, les stagiaires font souvent preuve d'une grande innocence quant à la valeur du dire. Dans les corpus utilisés apparaissent les représentations métalinguistiques des élèves. Les stagiaires se rendent alors compte que les représentations peuvent être modifiées profondément selon les questions et le guide donnés par l'enseignant.

En conclusion, l'objectif principal de ce mémoire est de remettre en question le caractère déterminant de leur discours sur la langue et de les amener à acquérir une certaine modestie.

- Les mémoires vont-ils être en ligne?

Ils vont l'être. Ils sont d'un intérêt inégal.

- Quelle est la chronologie de la formation en ce qui concerne les mémoires?

Les formateurs concernés par les mémoires en ont une dizaine à diriger chacun. Nous donnons aux stagiaires une bibliographie réduite en début d'année, ainsi qu'un cadrage général et un résumé des problématiques de mémoires présentés les années antérieures. Nous leur demandons également de prendre contact avec leur directeur.

51 Nous avons remarqué que l'on pouvait parler de plusieurs « périodes » dans la conception du mémoire : la panique au début, l'agressivité au mois de janvier et les premiers contacts pris au mois de février.Une bonne moitié des stagiaires est très enthousiaste et ils ont trouvé que le travail sur ce mémoire a amélioré leurs relations avec leurs élèves qui ont eu l'impression d'être vraiment pris en considération.Un tiers d'entre eux a fait quelque chose de moyen : ces stagiaires-là ont écrit ce qu'ils croient qu'on attend eux.

On retrouve à peu près tous les ans la même situation. De plus, il y a un discours ambiant qui dit que le mémoire n'est pas important.

- Quelle est l'importance du mémoire dans la validation?

3 Aucune personne dont le mémoire posait problème n'a jamais été validée. Certains ont dû refaire leur mémoire. De plus, l'inspection soutenait cette démarche jusqu'à présent.

- Le choix du sujet est-il libre?

En principe, les stagiaires arrivent avec des idées et il suffit de les affiner avec eux. Ils ne peuvent pas arriver en début d'année avec quelque chose de bien déterminé. Ils arrivent avec une formulation sur ce qu'ils maîtrisent.Parfois, nous rencontrons des stagiaires qui nous proposent des sujets décalés, par exemple, sur la motivation. Il faut donc les accompagner et les guider. Il y a un gros processus expérimental.

- Quel type de corpus recueillent les stagiaires pour les mémoires sur les rapports graphie phonie?

Les Suisses qui ont travaillé sur la lecture proposent des expérimentations en langue maternelle. Les stagiaires se fabriquent des protocoles d'expérimentation pour leurs classes à partir de cela. On peut leur donner envie mais en 6 mois ils ont peu de temps.

- Existe-t-il une formation à la direction de mémoires ? 

suivi des mémoires ne sont pas dans les établissements des stagiaires. Cette formation a été élargie à des tuteurs « de choc » qui sont aussi formés par leur stagiaire. Cela permet de faire une interaction entre les stagiaires et les tuteurs. Certains ont été intégrés cette année dans la soutenance des mémoires. Il y a un noyau de tuteurs fixe sur lesquels on peut s'appuyer. Les jurys de mémoires sont composés de 2 personnes : un assesseur et un directeur. Les tuteurs ont été assesseurs cette année.

- Un mémoire en transversal est-il possible?

Oui. Il faut néanmoins choisir des priorités. Le travail sur l'acquisition d'une langue est tellement difficile et long!

- Comment se déroule la soutenance?

Certains présentent ce qu'ils font, d'autres préfèrent que nous leur posions des questions.

\subsubsection{Conclusion}

Le but final de ce type de mémoire est de faire ressortir ce que les stagiaires ont dans la tête. Nous avons remarqué que pour savoir si les stagiaires ont appris quelque chose dans ce domaine, il ne faut pas leur poser la question directement car les réponses ne seront pas toujours fiables. Il faut essayer de poser des questions connexes. Et cette remarque a été également faite par les stagiaires sur leurs élèves et leurs apprentissages.

\subsection{La problématisation des supports dans la formation PLC}

Intervention de Roland Fischer (IUFM de Montpellier).

\subsubsection{Deux exemples concrets}

61 1. L'examen des pièces de monnaie en euros allemandes et françaises.

Les participants ont dû décrire ce qu'ils voyaient sur les deux faces des pièces allemandes et françaises qui leur ont été distribuées.Ensuite, nous sommes passés à une phase de conceptualisation : comment peut-on interpréter ces éléments graphiques?

On peut remarquer que l'on a quelque chose de concret au départ (les pièces) et que l'on arrive à quelque chose qui tient de l'abstrait. On a donc affaire à une symbolisation sur les pièces de monnaie, à une conceptualisation. On est en présence de diverses allégories (la république...) qui ont des paramètres constitutifs concrets (Marianne - la porte de Brandebourg).on peut donc se demander comment fonctionne ce transfert?

63 2. Étude rapide de deux poèmes, Der Panther de Rilke et L'Albatros de Baudelaire.

Après une étude brève et guidée des deux poèmes les participants ont pu faire la remarque suivante.Même si l'on ne possède que peu la langue utilisée dans ces poèmes (exemple ici, l'allemand pour le poème de Rilke), on se rend très vite compte que l'on a affaire à deux fonctionnements différents : Rilke travaille plus sur l'implicite. La clé du poème n'est pas donnée. Pour Baudelaire, on peut parler de comparaison car il travaille plus sur l'explicite. 


\subsubsection{Conclusion : quel lien avec l'enseignement des langues?} concrets peut être faite. Ainsi :

- Les deux exemples choisis ci-dessus portaient sur l'analyse contrastive. La problématisation des supports consiste en partie à essayer de trouver des éléments à mettre en perspective contrastive. Grâce à ce contraste, on met à jour des choses importantes.

- Dans l'enseignement des langues, il ne faut pas avoir peur d'élever le débat, tout en restant dans la logique de la perspective donnée par les manuels.

- Il ne faut pas amoindrir l'importance de l'interculturel, de la transversalité : il faut faire émerger des savoirs et des savoir-faire transférables ailleurs. La langue devient un instrument pour acquérir des nouveaux savoirs et savoir faire.

- À partir d'un point, donner aux élèves une perspective de ce qui pourrait être fait. Il est important d'utiliser des contournements plutôt que de poser des questions directes à l'élève. Si on prend une perspective trop analytique, on va ennuyer les élèves. Il faut passer par les émotions, jouer sur le côté affectif.

Cette démarche contrastive peut être également utilisée en primaire car cela permet d'ouvrir à l'altérité. La notion de l'absence de domaines réservés permet d'intéresser tous les élèves.

\section{Éléments de conclusion}

Les participants, devant la priorité nationale donnée aux langues, ont souhaité renouveler cette action PSE l'année prochaine. Il serait cependant souhaitable que la deuxième phase de cette action se déroule au mois de janvier plutôt sur le site de Nîmes qui est plus central. La langue étant une priorité, il serait nécessaire de prévoir des rencontres chaque année. Il serait de même souhaitable d'élargir ce dispositif aux autres IUFM de France pour une mutualisation et harmonisation réelles. L'absence d'équipes pédagogiques au sein d'un même IUFM peut peut-être être compensée par les rencontres PSE. L'existence d'une mailing listserait souhaitable.

En effet, il est apparu indispensable aux formateurs présents que la formation aille vers des contenus communs même et surtout entre les différents sites, le problème étant bien évidemment le manque de temps pour l'harmonisation.Enfin, on se doit de réagir en fonction des textes ministériels : priorité et diversification sont les deux termes associés à l'enseignement des langues. Il faut donc bien montrer cela dans les faits dans nos instituts. Cela passe en priorité par des créations de poste: l'existence matérielle de postes amènera une prise en considération supérieure des langues.Le problème vient aussi de ce que l'IUFM n'est pas le seul à intervenir dans la formation et il faudrait qu'il y ait rencontres et mutualisation entre les différents acteurs dans la formation. 
Moderne Française (collection : Brochures d'Éducation Nouvelle Populaire), № 79, Janvier 1953, p.

7.

FREINET C. et al. : La génèse de l'Homme. Cannes, Éditions de l'École

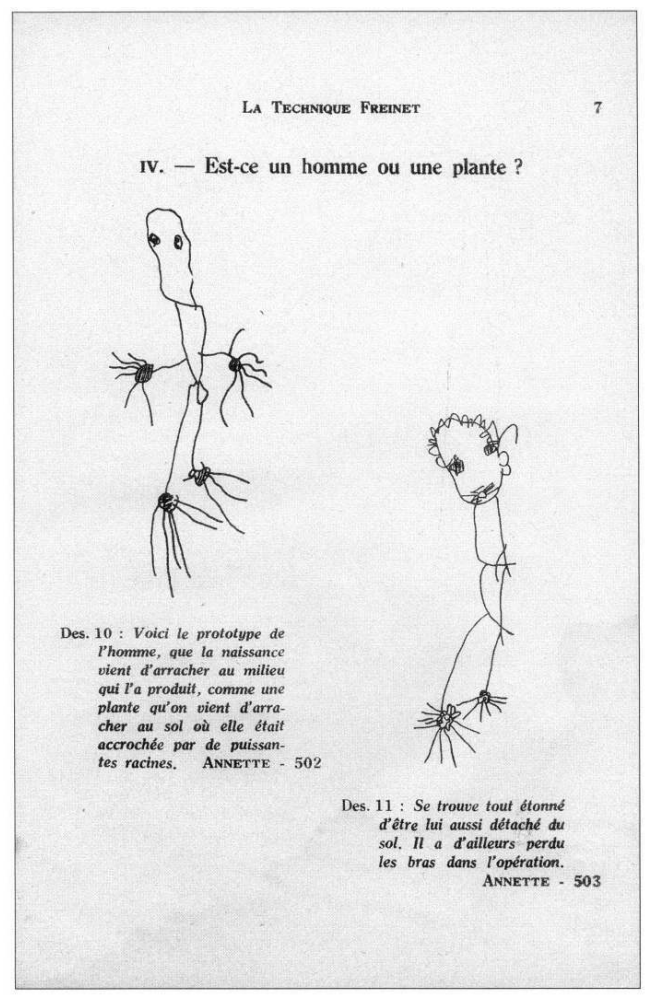

BIBLIOGRAPHIE

\section{Bibliographie sélective à l'usage des formateurs}

Suggestions bibliographiques concernant la notion du genre en éducation

BO N ${ }^{\circ} 10$ du 9 mars 2000 : Convention interministérielle et européenne pour la promotion de l'égalité des chances entre les filles et les garçons, les femmes et les hommes dans le système éducatif.

BO Nº 10 du 2 novembre $2000:$ A l'école, au collège et au lycée : de la mixité à l'égalité.

BAUDELOT C. et ESTABLET R. : Allez les filles ! Paris, Éditions du Seuil, 1992. BLANCHARD C. (dir.) : Variations sur une leçon de mathématiques, analyse d'une séquence, l'écriture des grands nombres . Paris, L'harmattan, 1997. 
BOUCHARD P. et ST AMAND J.-C. : Garçons et filles, stéréotypes et réussite scolaire. Montréal (Québec, Canada), Éditions du remue ménage, 1996.

CLAIR R. : La formation scientifique, un enseignement au-dessus de tout soupçon ? Liris, UNESCO, 1995.

CNRS, Inalf : Femme j'écris ton nom... Guide d'aide à la féminisation des noms de métiers, titres, grades et fonctions. Paris, La documentation française, 1999.

DURAND-DELVIGNE A. : « Sexisme par négligence », in Le Monde de l'éducation, Nov 1999.

DURU-BELLAT M. : L'école des filles, quelle formation pour quels rôles sociaux ? Paris, L'harmattan, 1990.

FONTANINI C. : Les filles face aux classes de mathématiques supérieures et spéciales : analyse des déterminants des choix d'une filière considérée comme atypique à leur sexe (direction : Marie Duru-Bellat). Université de bourgogne, 1999.

CALISSON R. : «À la recherche de l'éthique dans les disciplines d'intervention », in ELA, N 109, janv-mars 1998.

CALISSON R. : « Problématique de l'éducation et de la communication interculturelles en milieu européen », in $E L A, N^{\circ}$ 106, avril-juin 1997.

GRIFFATON A. : « Images des femmes dans les manuels d'anglais », in Les cahiers pédagogiques, $\mathrm{N}^{\circ}$ 372, mars 1999, p. 45.

HANNAFIN J. : "Co-education and attainment, a study of the gender effects of mixed and single-sex schooling on examination performance, Ph.D. dissertation" (thèse de doctorat), Université de Limerick, Limerick (Irlande), 1992.

LELIEVRE F. et LELIEVRE C. : Histoire de la scolarisation des filles. Paris, Nathan, 1991.

OREISTEIN P. : "School girls, Young Women, Self esteem and the confidence gap". Co-éd. : Anchor books - Doubleday, 1994.

Rapport de L'AAUW (association américaine des femmes universitaires) : “How schools shortchange girls". Marlowe et Company, 1995.

RIGNAUT S. et RICHERT P. : La représentation des hommes et des femmes dans les livres scolaires, rapport au premier ministre. Paris, La documentation française (collection des rapports officiels), 1997.

SADKER M. et SADKER D. : "Failing at fairness, How our schools cheat girls". Touchstone Editions, 1995.

SUNDERLAND J. : “Girls being quiet : aproblem for foreign language classrooms ?”, in Language Teaching Research. 2, 1, 1998, pp. 48-82.

SUNDERLAND J. : "Issues of language and gender in second and foreign language - Education", in Language Teaching. Octobre 2000, 33/4.

SUNDERLAND J. : "New understanding of gender and language classroom research texts, teacher talk and student talk", in Language Teaching Research. 4, 2, 2000, pp. 149-173.

VIDAL c. : « Le cerveau, le sexe et les maths », in Tangent, N 83, nov-déc 2001, p. 6.

ZAIDMAN C. : La mixité à l'école primaire. Paris, l'Harmattan (bibliothèque de féminisme), 1996. 


\section{Suggestions bibliographiques concernant la conception du métier d'enseignant et de la formation des professeurs des écoles?}

BAILLY D. : Didactique de l'anglais (1) Objectifs et contenus de l'enseignement. Paris, Nathan, 1997. LANG V. : La professionnalisation des enseignants. Paris, PUF, 1999.

PERRENOUD P. : « Préparer au métier d'enseignant, une formation professionnelle comme les autres? », in BOURDONCLE R. et DEMAILLY L. (éd.), Les professions de l'éducation et de la formation. Villeneuve d'Ascq, Presses Universitaires du Septentrion, 1998, pp. 175-191.

GAONAC'H D. : «L'enseignement précoce des langues étrangères 》, in Sciences humaines, $\mathrm{N}^{\circ} 123$, 2002, pp. 16-20.

AUDIN L., LIGOZAT M.A. et LUC C : Enseignement des Langues Vivantes au CM2. Paris, INRP, 1999.

LUC C. et BAILLY D. : Approche d'une langue étrangère à l'école, Vol. 1 et 2. Paris, INRP, 1992.

\section{Suggestions bibliographiques concernant Le mémoire PLC2}

BAILLY D. : L'acquisition de la détermination nominale, Vol. 2. Ophrys, 1990.

BAILLY D. : Didactique de l'anglais (1) Objectifs et Contenus de l'enseignement. Paris, Nathan, 1997.

BAILLY D. : Didactique de l'anglais (2) Mises en Euvre. Paris, Nathan, 1998.

BERTHOUD A.-C. : Activités métalinguistiques et acquisition d'une langue seconde. Etude des verbes déictiques allemands. Berne (Suisse), Peter Lang, 1982.

BERTHOUD A.-C. : «Les erreurs de l'apprenant au panier ou sous le microscope ? », in Langues Modernes, $\mathrm{N}^{\circ}$ 5, 1987, pp. 11-17.

BERTHOUD A.-C. et MONDADA L. : « Entrer en matière dans l'interaction verbale : Acquisition et co-constraction du topic en L2 ", in AILE, N 1, 1992, pp. 107-142.

BRONCKART J.P. : Les sciences du langage : un défi pour l'enseignement ? Rapport à l'UNESCO. Neuchâtel (Suisse), Delachaux et Niestlé, 1985.

BRONCKART J.P. : « L'acquisition des valeurs des temps des verbes. Etude comparative de l'allemand, du basque, du catalan, du français et de l'italien ", in Langue française, $\mathrm{N}^{\circ} 97,1993$, pp. 102-126.

BRONCKART J.P. : Activités langagières, Textes et Discours. Neuchâtel (Suisse), Delachaux et Niestlé, 1996.

GAONAC'H D. : Théories de l'apprentissage et acquisition d'une langue étrangère. Didier, 1987.

GAONAC'H D. (Éd.) : " Acquisition et utilisation d'une langue étrangère, l'approche cognitive ", in Numéro spécial du Français dans le Monde. Paris, Hachette, 1990.

GAONAC'H D. : « Contrôle et automatisation dans le fonctionnement en langue étrangère ", in BRIANE C. et CAIN A., Quelles perspectives pour la recherche en didactique des langues? Paris, INRP, 1995, pp. 67-72.

GAONAC'H D. : « Faut-il apprendre les langues étrangères le plus tôt possible ? ", in Les langues à l'école : un apprentissage ?, Haas, 1997. 
GIACOBBE J. : Acquisition d'une langue étrangère. Cognition et Interaction. Editions du CNRS, 1992. GIACOBBE J. : « Construction des formes lexicales et activité cognitive dans l'acquisition du français L2 ", in AILE, N³, 1994, pp. 29-54.

TREVISE A. : « Métalangue des manuels et représentations métalinguitiques des apprenants », in Bulletin d'information sur les manuels scolaires, $\mathrm{N}^{\circ} 24,1992$, pp. 30-36.

TREVISE A. : « La gestion cognitive de l'étrangeté dans l'acquisition d'une langue étrangère », in AILE, Nº 1, 1992, pp. 87-106.

TREVISE A. : Éléments de description de l'acquisition d'une langue étrangère, Thèse de doctorat d'état, Université de Paris VII, Ex. ronéoté, 1992.

TREVISE A. : « Acquisition/apprentissage/enseignement d'une langue 2 : modes d'observations, modes d'intervention ", in Études de Linguistique Appliquée, N 92, 1993, pp. 51-64.

TREVISE A. : « Réflexion, réflexivité et apprentissage des langues », in AILE, Nº 8, 1996, pp. 5-40.

TREVISE A. : « Métalexique, métadiscours et interactions métalinguistiques », in LINX, № 36, 1997, pp. 41-54.

\section{NOTES}

1. DURAND-DELVIGNE : 1999.

2. PERRENOUD : 1998.

3. LANG : 1999 .

\section{RÉSUMÉS}

L'enseignement des langues étant devenu une priorité nationale dans l'enseignement en France, il semble évident que cet enseignement est voué à jouer un rôle important dans la formation des enseignants dans les années à venir. Il apparaissait donc indispensable d'organiser un séminaire regroupant les formateurs en langues des divers IUFM pour qu'un échange de pratiques et une mutualisation efficaces puissent avoir lieu. L'harmonisation des contenus de formation est un passage obligé si nous voulons réussir à aider les futurs enseignants à devenir capables d'enseigner une langue vivante. Plusieurs thèmes très variés furent abordés au cours de ce séminaire et notamment la notion de genre, la généralisation de l'enseignement des langues en primaire et les nouvelles conceptions et du métier et de la formation qui l'accompagnent. Des comparaisons entre différentes approches de la formation et même différents systèmes (britannique et français) furent proposées également. Enfin, les résultats d'expériences menées sur le mémoire PLC et la problématisation des supports furent exposés et discutés.

Teaching foreign languages in primary school is one of the governmental priorities in France. Logically it is bound to play a great role in teacher training in the years to come. So it seemed important to organize a meeting between teachers involved in foreign language teaching in the different IUFM. Exchanging ideas and information seems to be a necessity if we want to succeed in helping future teachers to be able to teach a foreign language. Many themes were discussed, 
such as the problem of gender in teaching and training, the introduction of foreign language teaching in primary school as source of a new conception of teaching and training. In the same way, comparisons were made between several approaches in teacher training or several systems: teacher training in two different IUFM or in two different countries, Great Britain and France. Finally, the results of such experiments as "le mémoire PLC" or how to adapt documents to teaching were given to the participants and discussed.

\section{AUTEUR}

SABINE ULRICH

IUFM de Nîmes 